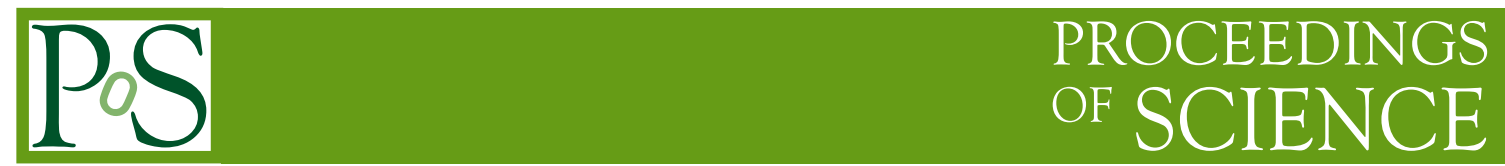

\title{
Non-perturbative (Lattice) QCD in B Physics
}

\author{
Elvira Gámiz* \\ CAFPE and Depto. de Física Teórica y del Cosmos, Universidad de Granada, E-18002, \\ Granada, Spain \\ E-mail: megamiz@ugr.es
}

Heavy quark quantities are useful for testing lattice techniques against well known experimental results, as well as for testing the Standard Model (SM), and searching for physics beyond the SM. I review the most recent lattice calculations on the $B$ sector relevant for this program including those of decay constants, semileptonic decay form factors, neutral $B$ mixing, and rare decays. I discuss the impact of recent improvements such as the use of relativistic actions to describe the $b$ quarks and simulations at the physical point.

14th International Conference on B-Physics at Hadron Machines, April 8-12, 2013

Bologna, Italy

* Speaker. 


\section{Introduction}

The relevance of lattice calculations of quantities involving $B$ mesons is derived not only from their contribution to the extraction of Standard Model (SM) parameters with high precision, but also from their potential to unveil New Physics (NP) effects and put constraints on Beyond the Standard Model (BSM) theories. This program must be carried out together with and having in mind existing and future experimental measurements. Experimental errors of most of the relevant quantities are now at the few percent level, we thus need determinations of the weak matrix elements involved in those processes with matching precision.

Accuracy in lattice calculations requires control over all the sources of systematic error. In particular, it is essential to take into account vacuum polarization effects in a realistic way, i.e., including up, down and strange sea quarks on the gauge configurations' generation. The up and down quarks are usually taken to be degenerate, so those simulations are referred to as $N_{f}=2+1$. Two lattice collaborations (FNAL/MILC and ETMC) are now generating configurations which also include the effects of charm quarks on the sea, $N_{f}=2+1+1$. The first preliminary results for flavour quantities on those configurations are starting to appear as described in these proceedings.

In the next Sections I will discuss the latest results for non-perturbative quantities relevant for $B$ physics from lattice QCD calculations with all sources of systematic error addressed. Among other things, that means that I will focus on simulations with $N_{f}=2+1$ and $N_{f}=2+1+1$ sea quarks.

\subsection{Heavy quarks on the lattice}

Simulating heavy quarks on the lattice implies having to deal with discretization errors entering in powers of the mass in lattice units, $a m_{Q}$. These corrections are not negligible at typical lattice spacings $a$. For $c$-quarks, the best strategy is improving the lattice actions to suppress $\left(a m_{Q}\right)^{n}$ corrections and thus keep those corrections under control. Following this strategy, HPQCD is using the HISQ formulation, especially designed to describe charm physics, and ETMC is using the twisted mass (tm) and the Osterwalder-Seiler (OS) formulations. The $b$-quark, however, can not be simulated with its physical mass on present lattices even with improved actions, since, typically, $a m_{b}>1$. There are two approaches that have been used to solve this problem: simulating the heavy quarks with effective theories and performing relativistic simulations with improved actions but with smaller masses than the physical bottom mass (but the same order or larger than the charm mass) and then extrapolate those results up to the physical $m_{b}$. The first approach is being used by the Alpha (heavy-quark effective theory, HQET), HPQCD (non-relativistic QCD, NRQCD), FNAL/MILC (Fermilab action), and RBC/UKQCD (non-peturbatively relativistic heavy-quark action) ${ }^{1}$ collaborations. One of the dominant systematic error in these calculations is the one associated with the use of an effective theory. The collaborations that follow a relativistic approach for $b$-quarks and whose results I will mention here are ETMC (tm action) and HPQCD (HISQ action).

\footnotetext{
${ }^{1}$ The Fermilab action starts with an improved Wilson action, which has the same heavy quark limit as QCD. With the Fermilab interpretation in terms of HQET this action can accurately describe $b$ and $c$ without errors that grow as $\left(a m_{Q}\right)^{n}$. The non-peturtatively relativistic heavy-quark action is a variant of the Fermilab action but with parameters tuned perturbatively.
} 


\section{Decay constants}

Unitarity Triangle fits are very sensitive to $f_{B}$ and processes with potential to reveal NP effects depend on $f_{B}$ or $f_{B_{s}}$, so any improvement in the decay constant calculations is very important.

Decay constants come from simple matrix elements, $\left\langle 0\left|A_{0}\right| B_{q}\right\rangle=M_{B_{q}} f_{B_{q}}$, which can be calculated on the lattice with high precision. The accuracy achieved is even higher for ratios of these quantities, since many systematic uncertainties and statistical fluctuations cancel partially or completely between numerator and denominator. There have been three lattice $N_{f}=2+1$ calculations of this parameter in the last two years by the HPQCD [1,2] and the FNAL/MILC [3] collaborations, which have significantly reduced previous errors. The HPQCD collaboration has also performed this year the first calculation including simulations at the physical values of the light quark masses and including effects from $c$ sea quarks [4]. The fact that the two most precise calculations of $f_{B_{s}}$, the ones in Ref. [1] and Ref. [4], that employ a relativistic and non-relativistic description of the $b$ quarks respectively and are statistically independent, are in a very good agreement $\left(f_{B_{s}}=224(4) \mathrm{MeV}\right.$ vs $\left.f_{B_{s}}=224(5) \mathrm{MeV}\right)$, is an excellent check of lattice techniques. The world averages, including the results in Refs. [1, 3, 4], are [4]

$$
f_{B^{+}}=185(3) \mathrm{MeV}, f_{B_{s}}=225(3) \mathrm{MeV}, \frac{f_{B_{s}}}{f_{B^{+}}}=1.218(8) .
$$

The result for $f_{B^{+}}$in Eq. (2.1) corresponds to the SM rate [4]:

$$
\frac{1}{\left|V_{u b}\right|^{2}} \mathscr{B} r\left(B^{+} \rightarrow \tau v\right)=6.05(20) \text {. }
$$

The direct comparison of Eq. (2.2) with experimental measurements of the $B$ leptonic decay width ${ }^{2}$ is problematic due to the need of the value of the CKM matrix element $\left|V_{u b}\right|$ (whose inclusive and exclusive determinations disagree at the $3 \sigma$ level) and the $\sim 2 \sigma$ disagreement of $\mathrm{BaBar}[5]$ and Belle's [6] measurements. Nevertheless, Belle new result seems to alleviate the tension between theory and experiment previously observed.

Ref. [4] also provides numbers for the charged and neutral modes

$$
f_{B^{+}}=184(4) \mathrm{MeV}, \quad f_{B^{0}}=188(4) \mathrm{MeV} .
$$

\section{Semileptonic decays}

\subsection{Extraction of CKM matrix elements $\left|V_{u b}\right|$ and $\left|V_{c b}\right|$}

There exist $\sim 2-3 \sigma$ tensions between the inclusive and the exclusive determinations of both $\left|V_{u b}\right|$ and $\left|V_{c b}\right|$. In addition to experimental measurements on, for example, $B \rightarrow \pi l v$ and $B \rightarrow$ $D\left(D^{*}\right) l v$, respectively, the exclusive determination of those CKM elements need as input form factors that can be calculated with high precision using lattice QCD techniques.

There have not been new lattice QCD calculations of the form factors describing the $B \rightarrow \pi l v$ decay since 2008 [7], although several analyses are in progress [8]. Using the lattice results in Ref. [7] and the latest experimental data [9], $\left|V_{u b}\right|_{\text {exclusive }}=(3.23 \pm 0.30) \cdot 10^{-3}$. A promising

\footnotetext{
${ }^{2}$ See the talk by Y. Horii's talk in this conference for a review on experimental measurements
} 
alternative for the exclusive extraction of $\left|V_{u b}\right|$ is the decay $B_{s} \rightarrow K l v$, whose branching fraction is expected to be measured by LHCb and BelleII. The form factors describing this process can be calculated on the lattice more accurately than those for $B \rightarrow \pi l v$ since the spectator quark is heavier, a $s$ quarks instead of a $d$. Preliminary results using a non-relativistic description of $b$ quarks can be found in [10].

For the exclusive determination of $\left|V_{c b}\right|$, the state-of-the art calculation of the relevant form factors is the FNAL/MILC analysis in [11], which studies the decay $B \rightarrow D^{*} l v$ at zero recoil. The updated result is [12] $\left|V_{c b}\right|=(39.54 \pm 0.50 \pm 0.74) \cdot 10^{-3}$, where the first error is experimental and the second one the uncertainty in the lattice calculation of the form factors. $\left|V_{c b}\right|$ can also be extracted from the process $B \rightarrow D l v$. However, in order to match the precision achieved with $B \rightarrow D^{*} l v$, one needs to study this process at non-zero recoil. The FNAL/MILC collaboration is doing an extensive study of both $B \rightarrow D^{*} l v$ and $B \rightarrow D l v$ decays at zero and non-zero recoil [13], providing two independent modes for the extraction of $\left|V_{c b}\right|$. This study will also provide checks of the shape of the form factors, in addition to $\left|V_{c b}\right|$.

For a more detailed discussion on the determination of $\left|V_{u b}\right|$ and $\left|V_{c b}\right|$ both exclusively and inclusively, see the talk by G. Ricciardi in these proceedings [12].

The FNAL/MILC collaboration recently analyzed a subset of their $B \rightarrow D l v$ data to calculate the ratio of branching fractions $R(D)=\mathscr{B} r(B \rightarrow D \tau v) / \mathscr{B} r(B \rightarrow D l v)=0.316(14)$ [14]. Their value is $\sim 1.7 \sigma$ smaller than the recent experimental measurement by the BaBar collaboration [15]. They found that the value of the ratio is very sensitive to differences in the scalar form factor, so one should be cautious in using indirect estimates of the form factors to constrain NP models in other decay channels such as $B \rightarrow D^{*} \tau \nu$ [14]. Given the present tensions, not only for $R(D)$ but for $R\left(D^{*}\right)$, and the possible indications of NP that could be extracted from a combined analysis of both set of decays, unquenched lattice QCD calculations of those two ratios should be a priority. Together with a determination of $\left|V_{c b}\right|$ and the shape of the form factors describing $B \rightarrow D\left(D^{*}\right) l v$ decays, the final FNAL/MILC analysis including the complete set of data will also provide an improved determination of $R(D)$ as well as a calculation of $R\left(D^{*}\right)$. Current experimental measurements of these ratios are statistics-limited, so Belle II and SuperB should significantly reduce the errors of those measurements.

\subsection{B rare decays}

There is an active effort [16] to constrain NP using experimental results for $B \rightarrow K l^{+} l^{-}$and other rare $B$ decays. After this conference, the HPQCD collaboration has published the first unquenched lattice QCD calculation of the form factors for $B \rightarrow K l^{+} l^{-}$in and beyond the SM [17]. In Ref. [18], the same authors report on $\mathscr{B}\left(B \rightarrow K l^{+} l^{-}\right)$in $q^{2}$ bins used by experiment, calculate ratios of branching fractions for $l=e, \mu, \tau$ and the "flat term" in the angular distribution, and compare the differential branching fractions $d \mathscr{B} / d q^{2}\left(B \rightarrow K l^{+} l^{-}\right)$with experimental measurements by Belle, BaBar, CDF, and LHCb. The FNAL/MILC collaboration is working on a similar project but using a different -although also based on an effective theory - description for the $b$ quarks. They present preliminary results from this analysis in Ref. [19].

Another process of interest for the studies in Refs. [16] is $B \rightarrow K^{*} l^{+} l^{-}$. The fact that there is an unstable vector meson in the final state complicates the analysis of this decay on the lattice and 


\begin{tabular}{cccc}
\hline \hline & HPQCD & FNAL/MILC & RBC/UKQCD \\
\hline$\xi$ & $1.258(33)$ & $1.27(6)$ & $1.13(12)$ \\
\hline$B_{B_{s}} / B_{B_{d}}$ & $1.05(7)$ & $1.06(11)$ & - \\
HPQCD: $f_{B_{s}} \sqrt{\hat{B}_{B_{s}}}=266(6)(17) \mathrm{MeV}$, & $\hat{B}_{B_{s}}=1.33(6)$ \\
HPQCD: $f_{B_{d}} \sqrt{\hat{B}_{B_{d}}}=216(9)(13) \mathrm{MeV}$, & $\hat{B}_{B_{d}}=1.26(11)$ \\
\hline \hline
\end{tabular}

Table 1: $B$-meson mixing parameters. $\xi$ is defined as the ratio of the parameters in the second and third rows. In the case where there are two errors, the first one is statistical and the second one systematic.

there is not an unquenched calculation of the relevant form factors yet. Work in progress with $2+1$ flavours of sea quarks by the Cambridge group can be found in Ref. [20].

\section{Neutral $B-$ meson mixing}

It has been argued that differences observed between measurements of some flavor observables and the corresponding SM predictions may be due to BSM physics affecting the neutral $B$-meson mixing processes [21, 22]. Although the most recent analysis [23, 24] and measurements [25] seem to indicate that there are not large BSM contribution to neutral $B$-meson mixing, the future will bring new twists, and precise calculations of the non-perturbative inputs parametrizing the mixing in the SM and beyond are necessary for a thorough understanding of quark flavor physics. The current status of $N_{f}=2+1$ lattice calculations of the non-perturbative quantities parametrizing the mass differences between the heavy and the light mass eigenstates in both the $B_{d}^{0}$ and $B_{s}^{0}$ systems, as well as the $\mathrm{SU}(3)$ breaking ratio $\xi$, is summarized in Tab. 1. The HPQCD [26] and FNAL/MILC [27] collaborations use the same light quark formulation, but a different description for the $b$ quarks. The exploratory study by the RBC/UKQCD collaboration uses heavy quarks in the static limit [28]. The average of the results in Tab. 1 for $\xi$ gives the value $\xi=1.251 \pm 0.032$. The calculations whose results are shown in Tab. 1 were not optimized to extract the bag parameters but rather the matrix elements, so the errors for the bag parameters can be significantly reduced to about a $3 \%$ error in on-going calculations.

There is not yet a finalized calculation of the matrix elements needed for the determination of the decay width differences, $\Delta \Gamma_{d, s}$, in the continuum limit and with $N_{f}=2+1$ flavors of sea quarks, but preliminary results for the relevant matrix elements by FNAL/MILC can be found in [29].

Beyond the SM the mixing parameters can have contributions from $\Delta B=2$ four-fermion operators which do not contribute in the SM. The matrix elements of the five operators in the complete basis describing $\Delta B=2$ processes, together with the Wilson coefficients for those operators calculated in a particular BSM theory and the experimental measurements of the mixing parameters, can provide very useful constraints on that BSM theory. Again, there is not a final unquenched lattice calculation of the matrix elements of all the operators in the $\Delta B=2$ effective hamiltonian, but FNAL/MILC presented preliminary results for the complete basis in [29].

The authors of Ref. [30] suggested that the branching fractions of the rare decays $B_{q} \rightarrow \mu^{+} \mu^{-}$ (for $q=s, d$ ) could be determined from the experimental measurement of the mass difference in 
the neutral $B_{q}$-meson system, $\Delta M_{q}$, and the lattice calculation of the bag parameter $\hat{B}_{B_{q}}$ using

$$
\frac{\mathscr{B} r\left(B_{q} \rightarrow \mu^{+} \mu^{-}\right)}{\Delta M_{q}}=\tau\left(B_{q}\right) 6 \pi \frac{\eta_{Y}}{\eta_{B}}\left(\frac{\alpha}{4 \pi M_{W} \sin ^{2} \theta_{W}}\right)^{2} m_{\mu}^{2} \frac{Y^{2}\left(x_{t}\right)}{S\left(x_{t}\right)} \frac{1}{\hat{B}_{q}} .
$$

In order to compare experimental measurements and the theory predictions for the decay rate of $B_{s}^{0}$, one must include the effects of a non-vanishing $\Delta \Gamma_{s}$ [31]. This can be done in the SM by rescaling the theory prediction by $1 /\left(1-y_{s}\right)$, where $y_{s} \equiv \tau_{B_{s}} \Delta \Gamma_{s} / 2$ [31]. Multiplying Eq. (4.1) by this factor for the $B_{s}^{0} \rightarrow \mu^{+} \mu^{-}$decay and using the HPQCD determination of the bag parameters $\hat{B}_{B_{S}}=1.33(6)$ one gets [32]

$$
\begin{aligned}
\left.\mathscr{B} r\left(B_{s} \rightarrow \mu^{+} \mu^{-}\right)\right|_{y_{s}} & =(3.71 \pm 0.17) \times 10^{-9} \\
\mathscr{B} r\left(B_{d} \rightarrow \mu^{+} \mu^{-}\right) & =(1.03 \pm 0.09) \times 10^{-10}
\end{aligned}
$$

The direct calculation of these branching fractions has become competitive with the one in (4.2) [33] thanks to the recent improvements in the calculation of the $B$-meson decay constants on the lattice summarized in Sec. 2. Including the correction factor $1 /\left(1-y_{s}\right)$ for the $B_{s} \rightarrow \mu^{+} \mu^{-}$ decay rate one gets ${ }^{3}$.

$$
\begin{aligned}
\left.\mathscr{B} r\left(B_{s} \rightarrow \mu^{+} \mu^{-}\right)\right|_{y_{s}} & =(3.56 \pm 0.18) \cdot 10^{-9} \\
\mathscr{B} r\left(B_{d} \rightarrow \mu^{+} \mu^{-}\right) & =(1.01 \pm 0.10) \cdot 10^{-10}
\end{aligned}
$$

The agreement between the two set of numbers in (4.2) and (4.3) is excellent. This gives us confidence in the SM prediction for these branching fractions, and this confidence will increase when we have results for the bag parameters entering in (4.2) from the on-going lattice calculations described above. This is very important since LHC is approaching the SM predictions, with the first evidence for one of these two processes recently reported by LHCb [34]. The LHCb measurement [34], $\mathscr{B} r\left(B_{s} \rightarrow \mu^{+} \mu^{-}\right)=\left(3.2_{-1.2}^{+1.5}\right) \cdot 10^{-9}$, is consistent with the SM prediction in Eqs. (4.2) and (4.3).

For a more detailed discussion on the rare processes $B_{q} \rightarrow \mu^{+} \mu^{-}$and their impact in phenomenology, see the talks by W. Altmannshofer [35], J. Girrbach [36], and R. Knegjens [37] in these proceedings.

\section{Conclusions}

Lattice QCD calculations of non-perturbative parameters relevant for $B$ flavour phenomenology have achieved accuracies at the per-cent level for many key quantities. The agreement between results from different collaborations for the same quantities also allows an important check of lattice methods, although it is still limited to a few quantities and two collaborations. More of these checks will be possible in the near future when several collaborations finish their on-going analyses. For the next two years we expect new results from the FNAL/MILC, ETM, and RBC/UKQCD collaborations for decay constant, $B^{0}-\bar{B}^{0}$ mixing, and $B \rightarrow \pi l v$.

\footnotetext{
${ }^{3}$ The value for $\mathscr{B} r\left(B_{S} \rightarrow \mu^{+} \mu^{-}\right)$is taken from Ref. [32].
} 
The first $N_{f}=2+1$ results for meson masses and decay constants using a relativistic approach are very promising. The next step in this direction will be the calculation of more complicated quantities such as semileptonic form factors and neutral meson mixing parameters. The first calculations with data simulated directly at the physical light quark masses (instead at heavier ones) has also appeared in the last year. This will reduce the systematic errors associated with the chiral extrapolations, especially in quantities involving $B$ mesons, where light quarks are in the valence sector.

The reduction of the dominant sources of errors will make necessary for lattice calculation to include some uncertainties which were subdominant until now like isospin breaking, electromagnetic effects, or dynamical charm quarks. Another target for lattice calculations is going beyond gold-platted quantities and develop methods to calculate more demanding quantities (weak decay to resonances, non-local operators, ...).

\section{Acknowledgements}

The author's work is supported in part by the MICINN under Grants FPA2010-16696 and FPA2006-05294, and Ramón y Cajal program; by Junta de Andalucía under Grants FQM-101, FQM-330, and FQM-6552; and by European Commission under Grant No. PCIG10-GA-2011303781.

\section{References}

[1] C. McNeile, C. T. H. Davies, E. Follana, K. Hornbostel and G. P. Lepage [HPQCD Collaboration], Phys. Rev. D 85 (2012) 031503 [arXiv:1110.4510 [hep-lat]];

[2] H. Na, C. J. Monahan, C. T. H. Davies, R. Horgan, G. P. Lepage and J. Shigemitsu [HPQCD Collaboration], arXiv:1202.4914 [hep-lat];

[3] A. Bazavov et al. [Fermilab Lattice and MILC Collaboration], Phys. Rev. D 85 (2012) 114506 [arXiv:1112.3051 [hep-lat]].

[4] R. J. Dowdall et al. [HPQCD Collaboration], arXiv:1302.2644 [hep-lat].

[5] J. P. Lees et al. [BABAR Collaboration], arXiv:1207.0698 [hep-ex].

[6] I. Adachi et al. [Belle Collaboration], arXiv:1208.4678 [hep-ex].

[7] J. A. Bailey et al., Phys. Rev. D 79 (2009) 054507 [arXiv:0811.3640 [hep-lat]].

[8] C. M. Bouchard, G. P. Lepage, C. J. Monahan, H. Na and J. Shigemitsu, PoS LATTICE 2012 (2012) 118 [arXiv:1210.6992 [hep-lat]];

F. Bahr, F. Bernardoni, A. Ramos, H. Simma, R. Sommer and J. Bulava, PoS LATTICE 2012 (2012) 110 [arXiv:1210.3478 [hep-lat]];

T. Kawanai, R. S. Van de Water and O. Witzel, PoS LATTICE 2012 (2012) 109 [arXiv:1211.0956 [hep-lat]].

[9] Y. Amhis et al. [Heavy Flavor Averaging Group Collaboration], arXiv:1207.1158 [hep-ex].

[10] C. M. Bouchard, G. P. Lepage, C. J. Monahan, H. Na and J. Shigemitsu, PoS LATTICE 2012 (2012) 118 [arXiv:1210.6992 [hep-lat]]. 
[11] C. Bernard et al., Phys. Rev. D 79 (2009) 014506 [arXiv:0808.2519 [hep-lat]].

[12] G. Ricciardi, arXiv:1306.1039 [hep-ph].

[13] S. -W. Qiu, C. DeTar, D. Du, A. S. Kronfeld, J. Laiho and R. Van de Water, PoS LATTICE 2012 (2012) 119 [arXiv:1211.2247 [hep-lat]].

[14] J. A. Bailey et al., arXiv:1206.4992 [hep-ph].

[15] J. P. Lees et al. [BaBar Collaboration], arXiv:1205.5442 [hep-ex].

[16] D. Becirevic, N. Kosnik, F. Mescia and E. Schneider, Phys. Rev. D 86 (2012) 034034 [arXiv:1205.5811 [hep-ph]];

C. Bobeth, G. Hiller, D. van Dyk and C. Wacker, JHEP 1201 (2012) 107 [arXiv:1111.2558 [hep-ph]];

F. Beaujean, C. Bobeth, D. van Dyk and C. Wacker, JHEP 1208 (2012) 030 [arXiv:1205.1838 [hep-ph]];

C. Bobeth, G. Hiller and D. van Dyk, Phys. Rev. D 87 (2013) 034016 [arXiv:1212.2321 [hep-ph]].

[17] C. Bouchard, G. P. Lepage, C. Monahan, H. Na and J. Shigemitsu, arXiv:1306.2384 [hep-lat].

[18] C. Bouchard, G. P. Lepage, C. Monahan, H. Na and J. Shigemitsu, arXiv:1306.0434 [hep-ph].

[19] R. Zhou et al., PoS LATTICE 2012 (2012) 120 [arXiv:1211.1390 [hep-lat]].

[20] Z. Liu, S. Meinel, A. Hart, R. R. Horgan, E. H. Muller and M. Wingate, arXiv:1101.2726 [hep-ph].

[21] J. Laiho, E. Lunghi, R. Van De Water, PoS FPCP2010 (2010) 040 [arXiv:1102.3917 [hep-ph]];

[22] A. Lenz et al., Phys. Rev. D 83 (2011) 036004 [arXiv:1008.1593 [hep-ph]].

[23] A. Lenz et al., Phys. Rev. D 86, 033008 (2012) [arXiv:1203.0238 [hep-ph]].

[24] Talk by L. Silvestrini in this conference.

[25] M. Vesterinen [on behalf of the LHCb Collaboration], arXiv:1306.0092 [hep-ex].

[26] E. Gámiz et al. [HPQCD Collaboration], Phys. Rev. D 80 (2009) 014503 [arXiv:0902.1815 [hep-lat]].

[27] A. Bazavov et al., arXiv:1205.7013 [hep-lat].

[28] C. Albertus et al., Phys. Rev. D 82 (2010) 014505 [arXiv:1001.2023 [hep-lat]].

[29] C. M. Bouchard et al., PoS LATTICE 2011 (2011) 274 [arXiv:1112.5642 [hep-lat]].

[30] A. J. Buras, Phys. Lett. B 566 (2003) 115 [hep-ph/0303060].

[31] K. De Bruyn, R. Fleischer, R. Knegjens, P. Koppenburg, M. Merk and N. Tuning, Phys. Rev. D 86 (2012) 014027 [arXiv:1204.1735 [hep-ph]]; K. De Bruyn, R. Fleischer, R. Knegjens, P. Koppenburg, M. Merk, A. Pellegrino and N. Tuning, Phys. Rev. Lett. 109 (2012) 041801 [arXiv:1204.1737 [hep-ph]].

[32] A. J. Buras, R. Fleischer, J. Girrbach and R. Knegjens, arXiv:1303.3820 [hep-ph].

[33] A. J. Buras, J. Girrbach, D. Guadagnoli and G. Isidori, Eur. Phys. J. C 72 (2012) 2172 [arXiv:1208.0934 [hep-ph]].

[34] RAaij et al. [LHCb Collaboration], arXiv:1211.2674 [Unknown].

[35] W. Altmannshofer, arXiv:1306.0022 [hep-ph].

[36] J. Girrbach, arXiv:1305.4771 [hep-ph].

[37] R. Knegjens, arXiv:1305.6834 [hep-ph]. 